\title{
Impact of the Adaptive Statistical Iterative Reconstruction Technique on Radiation Dose and Image Quality in Bone SPECT/CT
}

\author{
Louis Sibille $^{1}$, Benjamin Chambert ${ }^{1}$, Sandrine Alonso ${ }^{2}$, Corinne Barrau ${ }^{1}$, Emmanuel D’Estanque $^{1}$, Yassine Al Tabaa ${ }^{1}$, \\ Laurent Collombier ${ }^{1}$, Christophe Demattei ${ }^{2}$, Pierre-Olivier Kotzki ${ }^{1}$, and Vincent Boudousq ${ }^{1}$
}

${ }^{1}$ Department of Nuclear Medicine, Nîmes University Hospital, Nîmes, France; and ${ }^{2}$ Department of Biostatistics (BESPIM), Nîmes University Hospital, Nîmes, France

\begin{abstract}
The purpose of this study was to compare a routine bone SPECT/CT protocol using CT reconstructed with filtered backprojection (FBP) with an optimized protocol using low-dose CT images reconstructed with adaptive statistical iterative reconstruction (ASiR). Methods: In this prospective study, enrolled patients underwent bone SPECT/CT, with 1 SPECT acquisition followed by 2 randomized $C T$ acquisitions: FBP CT (FBP; noise index, 25) and ASiR CT (70\% ASiR; noise index, 40). The image quality of both attenuation-corrected SPECT and CT images was visually (5-point Likert scale, 2 interpreters) and quantitatively (contrast ratio [CR] and signal-to-noise ratio [SNR]) estimated. The CT dose index volume, dose-length product, and effective dose were compared. Results: Seventy-five patients were enrolled in the study. Quantitative attenuation-corrected SPECT evaluation showed no inferiority for contrast ratio and SNR issued from FBP CT or ASiR CT (respectively, $13.41 \pm 7.83$ vs. $13.45 \pm 7.99$ and $2.33 \pm 0.83$ vs. $2.32 \pm 0.84)$. Qualitative image analysis showed no difference between attenuation-corrected SPECT images issued from FBP CT or ASiR CT for both interpreters (respectively, $3.5 \pm 0.6$ vs. $3.5 \pm 0.6$ and $3.6 \pm 0.5$ vs. $3.6 \pm 0.5$ ). Quantitative CT evaluation showed no inferiority for SNR between FBP and ASiR CT images (respectively, $0.93 \pm 0.16$ and $1.07 \pm 0.17$ ). Qualitative image analysis showed no quality difference between FBP and ASiR CT images for both interpreters (respectively, $3.8 \pm 0.5$ vs. $3.6 \pm 0.5$ and $4.0 \pm 0.1$ vs. $4.0 \pm$ 0.2). Mean CT dose index volume, dose-length product, and effective dose for ASiR CT $(3.0 \pm 2.0 \mathrm{mGy}, 148 \pm 85 \mathrm{mGy} \cdot \mathrm{cm}$, and $2.2 \pm$ $1.3 \mathrm{mSv})$ were significantly lower than for FBP CT $(8.5 \pm 3.7 \mathrm{mGy}$, $365 \pm 160 \mathrm{mGy} \cdot \mathrm{cm}$, and $5.5 \pm 2.4 \mathrm{mSv}$ ). Conclusion: The use of $70 \%$ ASiR blending in bone SPECT/CT can reduce the CT radiation dose by $60 \%$, with no sacrifice in attenuation-corrected SPECT and CT image quality, compared with the conventional protocol using FBP CT reconstruction technique.
\end{abstract}

Key Words: adaptive statistical iterative reconstruction (ASiR); SPECT/CT; bone scintigraphy; CT optimization; attenuation correction

J Nucl Med 2016; 57:1091-1095

DOI: 10.2967/jnumed.115.164772

Received Jul. 30, 2015; revision accepted Jan. 24, 2016.

For correspondence contact: Louis Sibille, Department of Nuclear Medicine, Nîmes University Hospital, Place du Pr. Robert Debré 30029 Nîmes Cedex 9, France.

E-mail: louis.sibille@chu-nimes.fr

Published online Mar. 24, 2016.

COPYRIGHT (C 2016 by the Society of Nuclear Medicine and Molecular Imaging, Inc.
D been applied in nuclear imaging since the early 1980 s $(1,2)$, their implementation in CT practice was limited by the small computational capacities at that time leading to cumbersome times of image formation. Development of high-performance computer processors allows, nowadays, the use of iterative algorithms in CT imaging. Each manufacturer has developed its own algorithm: Adaptive Statistical Iterative Reconstruction (ASiR; GE Healthcare, Inc.), iDose Iterative Model Reconstruction (iDose; Philips Healthcare, Inc.), Iterative Reconstruction in Image Space and Sinogram AFfirmed Iterative Reconstruction (IRIS and SAFIRE; Siemens Healthcare Solutions, Inc.), and AIDR 3D (Toshiba Medical Systems). More recent generations of iterative algorithms are now available on nonhybrid CT devices such as Model-Based Iterative Reconstruction (MBIR; GE Healthcare), Iterative Model Reconstruction (IMR; Philips Healthcare), and ADvanced Modeled Iterative REconstruction (ADMIRE; Siemens Healthcare Solutions). While maintaining the same image quality, iterative reconstructions reduce noise so that the tube current and the tube voltage can be reduced, thus reducing the dose. Many clinical studies supporting this hypothesis have been reported, describing the use of iterative reconstruction methods in abdomen, chest, head, and coronary CT (3). Nevertheless, to the best of our knowledge, the role of iterative reconstruction in SPECT/CT has so far never been investigated.

Bone SPECT is one of the most common nuclear medicine procedures. It is frequently performed with a hybrid SPECT/CT camera that can combine the functional imaging capabilities of SPECT with the precise anatomic overlay of CT images. It provides accurate attenuation-corrected and coregistered images and has been shown to improve sensitivity and specificity in the areas of malignant and benign bone disease (4-7).

Considering the potential risks of radiation-induced cancer due to low x-ray doses (8-10) and the ALARA (As Low As Reasonably Achievable) precautionary principle, dose reduction of SPECT/CT examinations is essential. The radiation dose delivered by CT can significantly increase the overall dose of SPECT/CT examinations. Thereby, the use of new CT iterative reconstruction, such as ASiR, to decrease CT dose radiation seems to be important if it does not reduce SPECT/CT image quality. ASiR is a first-generation iterative reconstruction method whose technical principles have been described elsewhere (11-13). Briefly, it is a hybrid reconstruction algorithm designed as a compromise between filtered backprojection (FBP) (approximate but highly efficient and very-short reconstruction times) and iterative (optimized for noise reduction but high 
computational burden) methods. The FBP image is used for the initial condition. The iterative part of the ASiR algorithm includes system statistics modeling to optimize image reconstruction but does not include the system optics modeling. Final result corresponds to a blend of the original FBP and the full adaptive statistical iterative reconstruction images that can be adjusted from 1\%-100\%. Thus, ASiR reconstructs images with lower image noise and only slightly longer reconstruction time than FBP techniques. The aim of the present study was to assess the effect of ASiR on dose reduction and image quality in bone SPECT/CT.

\section{MATERIALS AND METHODS}

\section{Patients and Settings}

This prospective, single-center, observational study was conducted at the Department of Nuclear Medicine of Nimes University. Patients were enrolled for a 10-mo period (August 2013 to May 2014). The study was approved by the institutional ethics committee (CPP Sud Mediterranée III; RCB no. 2013-A00070-45). Patients referred for bone scanning (oncologic indications or lumbar complaints) were prospectively recruited. Because of possible artifacts in attenuation-corrected bone SPECT/CT, patients with lumbar osteosynthesis were excluded. All patients gave written informed consent to be enrolled.

\section{Scanning Techniques}

All examinations were performed on a Discovery NM/CT 670 camera (GE Healthcare) that combines a dual-head SPECT system (0.952-cm NaI crystal; 59 photomultiplier tube [PMT]; 1 analog-to-digital converter/PMT; $40 \times 54 \mathrm{~cm}$ field of view) with the BrightSpeed Elite multidetector CT (24 rows - maximum 16 slices/rotation).

SPECT. The $10 \mathrm{MBq} / \mathrm{kg}$ dose of ${ }^{99 \mathrm{~m}} \mathrm{Tc}$-hydroxymethylene diphosphonate (HMDP) (Osteocis; Cis Bio International-IBA Molecular) was administered intravenously at least $2 \mathrm{~h}$ before imaging. Low-energy, ultra-highresolution (LEHR) parallel collimators were used. SPECT studies were performed with the followings parameters: LEHR parallel collimators, 2 energy windows (one for the photopeak of ${ }^{99 \mathrm{~m}} \mathrm{Tc}$ [140 $\left.\pm 7.5 \% \mathrm{keV}\right]$ and the other for Compton downscatter correction [120 $\pm 7.5 \% \mathrm{keV}]$ ), $128 \times 128$ matrix, 4.42-mm pixel size, and $6^{\circ}$ per steps (arc per detector $\left.180^{\circ}\right)$ in step-and-shoot acquisition mode ( $15 \mathrm{~s}$ per step).

Data were reconstructed using an ordered-subset expectation maximization iterative reconstruction algorithm (10 subsets and 4 iterations) with collimator-detector response compensation (Evolution for Bone; GE Healthcare), scatter correction, and attenuation correction. All data were reconstructed twice using either FBP CT or ASiR $\mathrm{CT}$ to create attenuation-correction maps. Images were postfiltered using the standard Butterworth filter (cutoff, 0.48; order, 10).

CT. Every examination started with an acquisition of 2 scout views, a lateral view, and an anteroposterior view, using $120 \mathrm{kV}$ and $10 \mathrm{~mA}$. Two consecutive and randomized unenhanced craniocaudal CT acquisitions were acquired using helical rotation (beam collimation, $16 \times 1.25 \mathrm{~mm} ; 27.5 \mathrm{~mm} /$ rotation; pitch, $1.375 / 1$; rotation, $0.8 \mathrm{~s}$; $120 \mathrm{kV}$; automatic tube current modulation in the $x$-axis, $y$-axis, and $z$-axis; SmartmA and AutomA [GE Healthcare] maximum range, 10$350 \mathrm{~mA}$; noise index [NI], 25 for FBP CT or 40 for ASiR CT with $70 \%$ level of ASiR-FBP blending; total exposure, $15 \mathrm{~s}$ ) and were reconstructed using a $512 \times 512$ matrix, 0.98 pixel size, 2.5 slice thickness, 1.250 interval reconstruction, bone filter, and extended field of view. CT protocols differed only by the NI used (FBP CT NI25 and ASiR CT NI40) during acquisition and by the algorithm used for reconstruction (FBP CT and ASiR CT with 70\% level of ASiR-FBP blending) (Table 1). For FBP CT, NI25 was used as by the manufacturer. For the ASiR CT protocol, we chose an optimal level of NI and ASiR percentage (NI40, ASiR 70\%) that seemed to lead to the best trade-off between dose reduction and sufficient image quality (with a margin of
TABLE 1

Protocols for Each Scanning Technique

\begin{tabular}{lcc}
\hline \multirow{1}{*}{ Protocol } & \multicolumn{2}{c}{$\begin{array}{c}\text { Discovery NM/CT 670 } \\
\text { (BrightSpeed Elite, } \\
\text { 16-slice CT scanner) }\end{array}$} \\
\cline { 2 - 3 } & FBP CT & ASiR CT \\
\hline Reconstruction algorithm & FBP & ASiR 70\% \\
\hline Noise index & 25 & 40 \\
\hline Auto mA tube current range $(\mathrm{mA})$ & $10-350$ & $10-350$ \\
\hline Tube voltage $(\mathrm{kV})$ & 120 & 120 \\
\hline Rotation time $(\mathrm{ms})$ & 0.8 & 0.8 \\
\hline Detector collimation $(\mathrm{mm})$ & $16 \times 1.25$ & $16 \times 1.25$ \\
\hline Pitch & 1.375 & 1.375 \\
\hline Slice thickness/interval $(\mathrm{mm})$ & $2.5 / 1.25$ & $2.5 / 1.25$ \\
\hline
\end{tabular}

safety) according to our phantom study and the following formula: $\mathrm{NI}_{\mathrm{ASiR}}=\mathrm{NI}_{\mathrm{FBP}} /(1-0.0073 \times \% \mathrm{ASiR})(14,15)$. Remember that the NI is a manufacturer-specific term, which is defined as the SD of the noise in the central region of a CT image of a uniform $20-\mathrm{cm}$ water phantom when imaged and reconstructed with a standard reconstruction algorithm. We did not evaluate the computational time of FBP and ASiR reconstructions, but extra time due to iterative reconstruction did not have any impact on workflow.

\section{Processing and Image Evaluation}

Quantitative Analysis. Quantitative analysis was performed on an Xeleris 3 workstation (GE Healthcare) using a customized application programmed with Aladdin (GE Healthcare) that was able to create exactly the same cylindric volume of interest (VOI) on 2 paired sets of images $\left(\mathrm{SPECT}_{\mathrm{FBP}}\right.$ with $\mathrm{SPECT}_{\mathrm{ASiR}}$ or FBP CT with ASiR CT). With $2 \mathrm{CT}$ acquisitions, each patient acted as his or her own control. Quantitative SPECT evaluation was performed by analyzing 2 cylindric VOIs of the same size $\left(\sim 1 \mathrm{~cm}^{3}\right)$ in the body of the third lumbar vertebra (L3) and in the corresponding abdominal aorta (Ao). The total counts within the VOIs were determined, and a contrast ratio $(\mathrm{CR})$ was calculated as follows: $\mathrm{CR}=\mathrm{VOI}_{\mathrm{L} 3} / \mathrm{VOI}_{\mathrm{Ao}}$.

For the aorta VOI, average counts (mean) and SD were also determined to calculate a signal-to-noise ratio (SNR): $\mathrm{VOI}_{\text {Aomean }} / \mathrm{VOI}_{\mathrm{AoSD}}$.

Quantitative CT evaluation was performed by analyzing cylindric VOIs $\left(\sim 1 \mathrm{~cm}^{3}\right)$ in the abdominal aorta, carefully avoiding the vascular wall because it could contain calcified atheromatous plaque. Average density and SD were measured using Hounsfield units, and an SNR was calculated as follows: $\mathrm{SNR}=\mathrm{VOI}_{\mathrm{Aomean}} / \mathrm{VOI}_{\mathrm{AoSD}}$.

Qualitative Analysis. Qualitative image analysis was performed by 2 senior nuclear medicine physicians (7 and $10 \mathrm{y}$ of experience). All SPECT/CT image datasets were reviewed in a masked manner on an Xeleris 3 workstation using volumetrix MI evolution for bone. Visual SPECT evaluation consisted of assessing contrast and noise in the images. Visual CT evaluation used bone (CT window/center, 2,000/400 HU) and soft-tissue (CT window/center, 350/40 HU) CT windows to assess contrast, resolution, and artifacts of trabecular/cortical bones and adjacent soft tissue. For both SPECT and CT, image quality was scored on a 5-point Likert scale (1, unacceptable image quality; 2, suboptimal image quality; 3 , acceptable image quality; 4 , good image quality; or 5, excellent image quality).

CT Dose Comparison. CT dose index volume $\left(\mathrm{CTDI}_{\mathrm{vol}}\right)$ and doselength product (DLP) were compared for FBP CT and ASiR CT examinations. The effective dose was estimated using DLP and an abdominal dose conversion coefficient of 0.015 according to an International Commission on Radiological Protection publication (16). 


\section{Statistics}

Statistical analysis was performed using statistical analysis system 9.4 (SAS Institute Inc.). The type I error was fixed at 0.05 . Noninferiority analyses were performed with a 1-sided Schuirmann test. In this crossover study, if the period effect was significant, noninferiority analyses were conducted by period. CT dose indices and effective dose were compared using the paired Wilcoxon/Mann-Whitney test.

\section{RESULTS}

\section{Patients}

From August 19, 2013, to May 28, 2014, 75 patients were recruited. The study population consisted of 37 women and 38 men (median age, $66 \mathrm{y}$; age range, 45-85 y) whose weight and height were measured and body mass index (BMI) estimated $\left(26.3 \mathrm{~kg} / \mathrm{m}^{2}\right.$; range, $19.1-$ $\left.48.8 \mathrm{~kg} / \mathrm{m}^{2}\right)$. Nononcologic indications $(n=16$ [21\%]) for bone scans were chronic or posttraumatic lower back pain. Oncologic indications ( $n=59$ [79\%]) were initial staging and follow-up. Primary tumors were prostate $(n=26)$, breast $(n=24)$, kidney $(n=3)$, bladder $(n=2)$, colon $(n=2)$, lung $(n=1)$, and pancreas $(n=1)$. Data from 3 patients ( $n=75$ ) could not be used because of patient movement between the 2 CT acquisitions, incomplete examination, and camera breakdown. Figure 1 shows SPECT and CT axial images obtained with FBP and ASiR CT for a representative patient. ${ }^{99 \mathrm{~m}} \mathrm{Tc}-\mathrm{HMDP}$ was administered intravenously $205 \mathrm{~min}$ (range, 120-327 $\mathrm{min}$ ) before imaging.

\section{Attenuation-Corrected SPECT Image Analysis}

Quantitative SPECT evaluation showed no inferiority for CR and SNR between FBP CT and ASiR CT, with a mean CR of $13.41 \pm$ 7.83 for FBP CT and $13.45 \pm 7.99$ for ASiR CT and a mean SNR of $2.33 \pm 0.83$ for FBP CT and $2.32 \pm 0.84$ for ASiR CT (Table 2). Qualitative image analysis showed no difference between SPECT images issued from FBP CT or ASiR CT, with an equal mean Likert score of $3.5 \pm 0.6$ and $3.6 \pm 0.5$ for interpreter 1 and interpreter 2 , respectively.

\section{CT Images Analysis}

Quantitative CT evaluation showed no inferiority for SNR between FBP and ASiR CT images, with a mean SNR of $0.93 \pm$ 0.16 for FBP CT and $1.07 \pm 0.17$ for ASiR CT (Table 3 ).

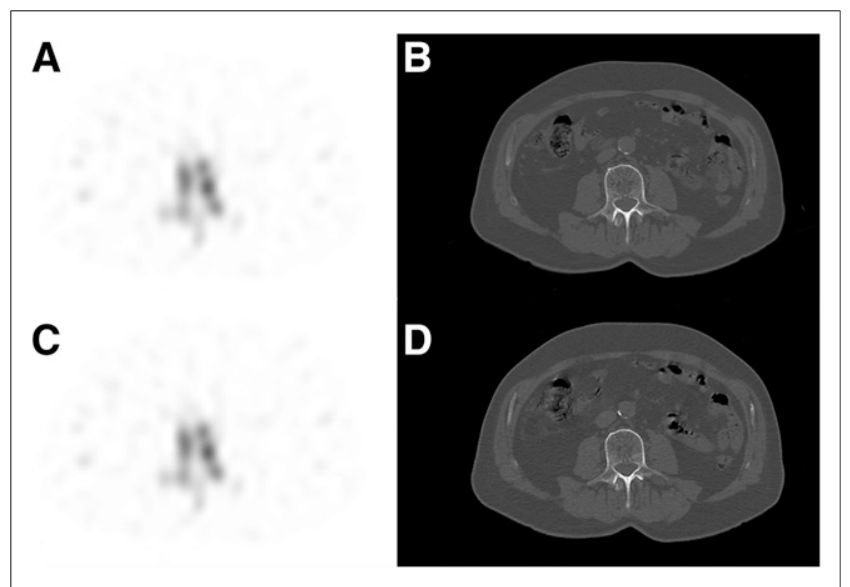

FIGURE 1. A 70-y-old man (height, $1.73 \mathrm{~m}$; weight, $71 \mathrm{~kg}$; body mass index, $24 \mathrm{~kg} / \mathrm{m}^{2}$ ) referred for prostate cancer staging bone scanning (in-

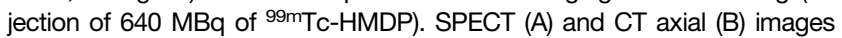
obtained with FBP CT (NI25; CTDI ${ }_{\text {vol }}, 6.4$ mGy; DLP, 276 mGy.cm; effective dose, $4.1 \mathrm{mSv}$ ). SPECT (C) and CT axial (D) images obtained with ASiR CT (NI40; CTDI ${ }_{\mathrm{vol}}, 2.5 \mathrm{mGy}$; DLP, $107 \mathrm{mGy} \cdot \mathrm{cm}$; effective dose, $1.6 \mathrm{mSv}$ ).
TABLE 2

SPECT Image Noise, Image Contrast, and Subjective Image Quality Score

\begin{tabular}{lrrr}
\hline Quality index & FBP SPECT & ASiR SPECT & $\begin{array}{c}P \\
\text { (noninferiority) }\end{array}$ \\
\hline Likert no. 1 & $3.5 \pm 0.6$ & $3.5 \pm 0.6$ & $<0.0001$ \\
Likert no. 2 & $3.6 \pm 0.5$ & $3.6 \pm 0.5$ & $<0.0001$ \\
CR & $13.41 \pm 7.83$ & $13.45 \pm 7.99$ & $<0.0001$ \\
SNR & $2.33 \pm 0.83$ & $2.32 \pm 0.84$ & $<0.0001$ \\
& & & \\
\hline & & & \\
Likert = 5-point scale image quality score. & \\
Data are mean \pm SD.
\end{tabular}

Qualitative image analysis showed no difference between FBP and ASiR CT images, with a mean Likert score of $3.8 \pm 0.5$ and $4.0 \pm 0.1$ for FBP CT and 3.6 \pm 0.5 and $4.0 \pm 0.2$ for ASiR CT for interpreter 1 and interpreter 2 , respectively.

\section{CT Dose Comparison}

Mean CTDI $_{\text {vol }}$, DLP, and effective dose for ASiR CT (3.0 \pm $2.0 \mathrm{mGy}, 148 \pm 85 \mathrm{mGy} \cdot \mathrm{cm}$, and $2.2 \pm 1.3 \mathrm{mSv}$ ) were significantly lower than for FBP CT $(8.5 \pm 3.7 \mathrm{mGy}, 365 \pm 160 \mathrm{mGy} \cdot \mathrm{cm}$, and $5.5 \pm 2.4 \mathrm{mSv}$ ) (Table 4).

\section{DISCUSSION}

Bone scanning is one of the most common examinations performed in nuclear medicine and is routinely used in oncologic staging, especially in patients with prostate and breast cancers. SPECT/CT has increased sensitivity and specificity over SPECT and is more efficient in characterizing equivocal bone lesions $(5,17,18)$. Large coverage of a total area of 2 SPECT fields (from the cervical region to the proximal femora) allows accurate analysis of the whole spine, which is the most frequent site of bone metastasis $(19,20)$. A growing use of CT implemented on hybrid SPECT/CT cameras improved patient care but also raised the total radiation delivered. CT irradiation can reach more than half of the total effective dose of different nuclear medicine examinations (21). It is, therefore, essential to follow the principle of optimization (As Low As Reasonably Achievable [ALARA]) for both SPECT and CT components using solutions such as automatic exposure control with tube current modulation and newly implemented iterative image reconstruction.

Iterative reconstruction algorithms have been well studied on soft-tissue CT scans (3). However, few studies focused on bone

TABLE 3

CT Image Noise and Subjective Image Quality Score

\begin{tabular}{lccc}
\hline Quality index & FBP CT & ASiR CT & $P$ (noninferiority) \\
\hline Likert no. 1 & $3.8 \pm 0.5$ & $3.6 \pm 0.5$ & $<0.0001$ \\
Likert no. 2 & $4.0 \pm 0.1$ & $4.0 \pm 0.2$ & $<0.0001$ \\
SNR & $0.93 \pm 0.16$ & $1.07 \pm 0.17$ & $<0.0001$
\end{tabular}

Likert $=5$-point scale image quality score.

Data are mean \pm SD. 
TABLE 4

CT Radiation Dose Reduction

\begin{tabular}{lccc}
\hline \multicolumn{1}{c}{ CT dose index } & FBP CT & ASiR CT & $P$ \\
\hline CTDI $_{\text {vol }}(\mathrm{mGy})$ & $8.5 \pm 3.7$ & $3.0 \pm 2.0$ & $<0.0001$ \\
\hline DLP $(\mathrm{mGy} \cdot \mathrm{cm})$ & $365 \pm 160$ & $148 \pm 85$ & $<0.0001$ \\
Effective dose $(\mathrm{mSv})$ & $5.5 \pm 2.4$ & $2.2 \pm 1.3$ & $<0.0001$
\end{tabular}

Data are mean \pm SD.

tissue, for which the contrast-to-noise ratio is naturally high. In this case, the advantage of iterative reconstruction might be limited (22-24). Spatial resolution is of special concern in bone imaging. Use of iterative reconstruction tends to smooth the subjective appearance of images, but it seems not to affect the spatial resolution $(15,25,26)$.

Manufacturers commonly recommend an ASiR level of $40 \%$. Various studies have set different blends of ASiR from $20 \%$ to $100 \%$, with reported dose reductions ranging from $23 \%$ to $76 \%$ (3). In this study, we chose an optimal level of NI and ASiR percentage (NI40 and ASiR 70\%) that seemed to lead to the best trade-off between dose reduction and sufficient image quality according to our phantom study and the following formula: $\mathrm{NI}_{\mathrm{ASiR}}=\mathrm{NI}_{\mathrm{FBP}} /(1-0.0073 * \% \mathrm{ASiR})$ $(14,15)$. We decided not to use a percentage higher than $70 \%$ because this may result in a noise-free oversmooth artifactual image appearance, with a perceived lower image quality $(11,25)$.

CT parameters previously used (NI25 FBP) led to a sufficient image quality in addition to scintigraphic data to perform reliable diagnosis. Therefore, the aim of this study was not to improve CT image quality but to significantly reduce radiation dose. Using NI40 and ASiR 70\%, we demonstrated a $65 \%$ reduction of the mean $\mathrm{CTDI}_{\mathrm{vol}}$ and a $60 \%$ reduction of the mean effective dose without alteration of CT or SPECT image quality. Importantly, these results made us change our daily practice. Evaluations of oncology patients to detect bone involvement systematically include SPECT/CT images of the whole spine (2-3 SPECT fields). Optimization of all SPECT/CT and PET/CT procedures is essential to reduce radiation exposure. SPECT/CT scans that were individually patient-tailored also improved image quality.

Another approach of the utility of iterative reconstruction would have been to tip the scales in favor of image quality rather than dose reduction. CT and SPECT images in large patients are often of inferior quality because of excessive noise and artifacts. Methods used to maintain image quality (increasing tube current and potential, increasing injected radiotracer dose) are associated with higher radiation dose levels. Thus, obese patients benefit from noise reduction related to ASiR reconstruction (27-29). Studying CT or SPECT image quality differences (FBP ASiR), we did not find any relationship with BMI (data not shown). However, we designed this study to be as close as possible to our daily practice with a heterogeneous population; therefore, our study population included only $21(28 \%)$ patients with a BMI greater than or equal to 30 . Waist circumference was not measured to classify different types of obesity. We assume that another study focused on obese patients could have more valuable results to assess the impact of $\mathrm{ASiR}$ in this population.

There were some interobserver differences on visual SPECT image analysis: interpreter 1 used a Likert score of 2 for both SPECT images issued from FBP and ASiR CT for 3 patients whereas interpreter 2 used a score of 3; those patients mainly correspond to BMI greater than $30 \mathrm{~kg} / \mathrm{m}^{2}$. For CT image analysis, 1 patient with a BMI of $44.6 \mathrm{~kg} / \mathrm{m}^{2}$ had a Likert score of 2 (interpreter 1) and 3 (interpreter 2) for both FBP and ASiR CT images.

A limitation of our study is that the results we report apply to 1 SPECT/CT system (Discovery NM/CT 670 camera), a single iterative reconstruction method, and blending (70\% ASiR NI40) using only sharp (bone) convolution kernel.

These results cannot be extrapolated to second-generation iterative reconstruction techniques such as MBIR, which are not yet implemented on hybrid SPECT/CT systems. Second, this study was not designed to assess the impact of the ASiR algorithm on the diagnostic performance. Further, we did not study the impact of iterative reconstruction in a specific population such as obese patients or patients with high-density implants or prosthesis. Nevertheless, this is the first SPECT/CT study designed to clinically validate optimized CT acquisition and reconstruction with ASiR.

Finally, further study is needed to validate the optimal combination of NI and ASiR percentage when exploring soft tissues and using smooth kernels. Although such combinations have already been investigated in many CT studies (11,30-32), we may assume that those results cannot be directly extrapolated to SPECT/CT practice because equipment, protocols, and purpose are not the same. Moreover, few studies on the utility of iterative reconstruction to obtain ultra-low-dose CT attenuation maps in SPECT/CT and PET/CT have been conducted so far $(33,34)$.

\section{CONCLUSION}

The use of $70 \%$ ASiR blending in bone SPECT/CT can reduce CT radiation dose by $60 \%$, with no sacrifice in SPECT and CT image quality compared with the conventional FBP CT reconstruction technique.

\section{DISCLOSURE}

The costs of publication of this article were defrayed in part by the payment of page charges. Therefore, and solely to indicate this fact, this article is hereby marked "advertisement" in accordance with 18 USC section 1734 . No potential conflict of interest relevant to this article was reported.

\section{ACKNOWLEDGMENTS}

We thank Dr. Mariella Lomma for her help in editing assistance. The trial registration number is NCT01800084.

\section{REFERENCES}

1. Brooks RA, Weiss GH, Talbert AJ. A new approach to interpolation in computed tomography. J Comput Assist Tomogr. 1978;2:577-585.

2. Shepp LA, Vardi Y. Maximum likelihood reconstruction for emission tomography. IEEE Trans Med Imaging. 1982;1:113-122.

3. Willemink MJ, Leiner T, de Jong PA, et al. Iterative reconstruction techniques for computed tomography part 2: initial results in dose reduction and image quality. Eur Radiol. 2013;23:1632-1642.

4. Ghosh P. The role of SPECT/CT in skeletal malignancies. Semin Musculoskelet Radiol. 2014;18:175-193.

5. Mariani G, Bruselli L, Kuwert T, et al. A review on the clinical uses of SPECT/ CT. Eur J Nucl Med Mol Imaging. 2010;37:1959-1985.

6. Huellner MW, Strobel K. Clinical applications of SPECT/CT in imaging the extremities. Eur J Nucl Med Mol Imaging. 2014;41:S50-S58.

7. Scheyerer MJ, Pietsch C, Zimmermann SM, Osterhoff G, Simmen H-P, Werner CML. SPECT/CT for imaging of the spine and pelvis in clinical routine: 
a physician's perspective of the adoption of SPECT/CT in a clinical setting with a focus on trauma surgery. Eur J Nucl Med Mol Imaging. 2014;41:S59-S66.

8. Berrington de González A, Mahesh M, Kim K-P, et al. Projected cancer risks from computed tomographic scans performed in the United States in 2007. Arch Intern Med. 2009;169:2071-2077.

9. Pearce MS, Salotti JA, Little MP, et al. Radiation exposure from CT scans in childhood and subsequent risk of leukaemia and brain tumours: a retrospective cohort study. Lancet. 2012;380:499-505.

10. Smith-Bindman R, Lipson J, Marcus R, et al. Radiation dose associated with common computed tomography examinations and the associated lifetime attributable risk of cancer. Arch Intern Med. 2009;169:2078-2086.

11. Silva AC, Lawder HJ, Hara A, Kujak J, Pavlicek W. Innovations in CT dose reduction strategy: application of the adaptive statistical iterative reconstruction algorithm. AJR. 2010;194:191-199.

12. Singh S, Kalra MK, Gilman MD, et al. Adaptive statistical iterative reconstruction technique for radiation dose reduction in chest CT: a pilot study. Radiology. 2011;259:565-573.

13. Willemink MJ, de Jong PA, Leiner T, et al. Iterative reconstruction techniques for computed tomography part 1: technical principles. Eur Radiol. 2013;23:1623-1631.

14. Hara AK, Paden RG, Silva AC, Kujak JL, Lawder HJ, Pavlicek W. Iterative reconstruction technique for reducing body radiation dose at CT: feasibility study. AJR. 2009; 193:764-771.

15. Rampado O, Bossi L, Garabello D, Davini O, Ropolo R. Characterization of a computed tomography iterative reconstruction algorithm by image quality evaluations with an anthropomorphic phantom. Eur J Radiol. 2012;81:3172-3177.

16. Valentin J. Managing patient dose in multi-detector computed tomography (MDCT). ICRP Publication 102. Ann ICRP. 2007;37:1-79.

17. Ndlovu X, George R, Ellmann A, Warwick J. Should SPECT-CT replace SPECT for the evaluation of equivocal bone scan lesions in patients with underlying malignancies? Nucl Med Commun. 2010;31:659-665.

18. Sharma P, Singh H, Kumar R, et al. Bone scintigraphy in breast cancer: added value of hybrid SPECT-CT and its impact on patient management. Nucl Med Commun. 2012;33:139-147.

19. Krishnaney AA, Steinmetz MP, Benzel EC. Biomechanics of metastatic spine cancer. Neurosurg Clin N Am. 2004;15:375-380.

20. Palmedo H, Marx C, Ebert A, et al. Whole-body SPECT/CT for bone scintigraphy: diagnostic value and effect on patient management in oncological patients. Eur J Nucl Med Mol Imaging. 2014;41:59-67.

21. Brix G, Nekolla EA, Borowski M, Noßke D. Radiation risk and protection of patients in clinical SPECT/CT. Eur J Nucl Med Mol Imaging. 2014;41:S125-S136.

22. Becce F, Ben Salah Y, Verdun FR, et al. Computed tomography of the cervical spine: comparison of image quality between a standard-dose and a low-dose protocol using filtered back-projection and iterative reconstruction. Skeletal Radiol. 2013;42:937-945.

23. Geyer LL, Körner M, Hempel R, et al. Evaluation of a dedicated MDCT protocol using iterative image reconstruction after cervical spine trauma. Clin Radiol. 2013;68:e391-e396.

24. Omoumi P, Verdun FR, Ben Salah Y, et al. Low-dose multidetector computed tomography of the cervical spine: optimization of iterative reconstruction strength levels. Acta Radiol. 2014;55:335-344.

25. Ghetti C, Ortenzia O, Serreli G. CT iterative reconstruction in image space: a phantom study. Phys Med. 2012;28:161-165.

26. Noël PB, Fingerle AA, Renger B, Münzel D, Rummeny EJ, Dobritz M. Initial performance characterization of a clinical noise-suppressing reconstruction algorithm for MDCT. AJR. 2011;197:1404-1409.

27. Desai GS, Uppot RN, Yu EW, Kambadakone AR, Sahani DV. Impact of iterative reconstruction on image quality and radiation dose in multidetector CT of large body size adults. Eur Radiol. 2012;22:1631-1640.

28. Kligerman S, Mehta D, Farnadesh M, Jeudy J, Olsen K, White C. Use of a hybrid iterative reconstruction technique to reduce image noise and improve image quality in obese patients undergoing computed tomographic pulmonary angiography. J Thorac Imaging. 2013;28:49-59.

29. Schindera ST, Odedra D, Mercer D, et al. Hybrid iterative reconstruction technique for abdominal CT protocols in obese patients: assessment of image quality, radiation dose, and low-contrast detectability in a phantom. AJR. 2014;202: W146-W152.

30. Böning G, Schäfer M, Grupp U, et al. Comparison of applied dose and image quality in staging CT of neuroendocrine tumor patients using standard filtered back projection and adaptive statistical iterative reconstruction. Eur J Radiol. 2015;84: 1601-1607.

31. Padole A, Singh S, Lira D, et al. Assessment of filtered back projection, adaptive statistical, and model-based iterative reconstruction for reduced dose abdominal computed tomography. J Comput Assist Tomogr. 2015;39:462-467.

32. Vachha B, Brodoefel H, Wilcox C, Hackney DB, Moonis G. Radiation dose reduction in soft tissue neck $\mathrm{CT}$ using adaptive statistical iterative reconstruction (ASIR). Eur J Radiol. 2013;82:2222-2226.

33. Brady SL, Shulkin BL. Ultralow dose computed tomography attenuation correction for pediatric PET CT using adaptive statistical iterative reconstruction. Med Phys. 2015;42:558-566.

34. Matsutomo N, Nagaki A, Sasaki M. Validation of the CT iterative reconstruction technique for low-dose CT attenuation correction for improving the quality of PET images in an obesity-simulating body phantom and clinical study. Nucl Med Commun. 2015;36:839-847. 